\title{
Reconstruction of the superior sagittal sinus with silicone tubing
}

\author{
Jinguian Ma, M.D., Ph.D., Tianqiang Song, Ph.D., Wang Hu, Ph.D., Michael Edgar \\ Muhumuza, M.D., Wenping ZhaO, B.S., Shuyuan Yang, Ph.D., J Jingwen Bai, Ph.D., \\ and Haixian Yang, Ph.D. \\ Department of Neurosurgery, Tianjin Medical University, General Hospital; and The Center of \\ Electron Microscopy, Tianjin Medical University, Tianjin, People's Republic of China
}

\begin{abstract}
Object. The authors conducted a study to develop a safe and effective intracranial venous sinus reconstruction for extensive clinical use.

Methods. After resecting the superior sagittal sinus (SSS), it was reconstructed in eight dogs by performing either a tube-insertion technique or end-to-end anastomosis procedure, in both of which a thin-walled silicone tube was used for repair. The patency of the SSS reconstruction was observed on digital subtraction angiography and transcranial Doppler ultrasonography, preoperatively and at 1,2, 4, and 8 weeks postoperatively. Histological and ultrastructural changes were observed using light and electron microscopy.

In five dogs the reconstructed SSS was patent, in one it was narrowed, in one it was completely occluded at the proximal site of the anastomosis, and one dog escaped from the laboratory 1 week postoperatively. The authors found no evidence of any additional neurological deficits, signs of toxicity, or side effects. Histological and ultrastructural studies generally showed vascular endothelial proliferation. No thrombosis occurred in the inner surface, at the site of anastomosis, or in the lumen of silicone tube nor in the sagittal sinus at up to 8 weeks postoperatively.

Conclusions. The use of a thin-walled silicone tube as an artificial substitute for intracranial dural venous sinus reconstruction seems to be a valuable technique. The silicone tubes were found to have good biological compatibility, nonthrombogenic effects, and a high patency rate. The method was found to be simple and effective as well as practicable in the clinic for the short term (8 weeks). The authors emphasize that Phase I clinical trials involving silicone tube-assisted SSS reconstruction require further research.
\end{abstract}

KEY WORDS - superior sagittal sinus - silicone tube $\bullet$ reconstruction $\bullet \operatorname{dog}$

The purpose of this experiment is to provide evidence that the use of silicone tubes in SSS reconstruction can be safe and effective and that the technique may be important for clinical use in future.

Currently a number of materials such as autogenous great saphenous vein, neck superficial veins, artificial blood vessels (such as Dacron and polytetrefluoroethylene suitable for arterial systems of larger diameter), and silicone tubing (suitable for the dural venous sinuses and veins) are in use; however, the optimum material remains a subject of debate. Trauma-induced lesions and tumors (especially parasagittal meningioma) that invade the middle and posterior two thirds of the SSS often involve the venous sinus. It is often a necessary to reconstruct the SSS to avoid serious postoperative complications. The results of our experiment, similar to those reported by Sakaki, et al., ${ }^{8}$ indicate that silicone tubing can be an effective material in the reconstruction of dural venous sinuses and veins; however, long-term results need to be studied further clinically.

Abbreviations used in this paper: $\mathrm{SSS}=$ superior sagittal sinus; $\mathrm{TCD}=$ transcranial Doppler.

\section{MATERIALS AND METHODS}

\section{Animals and Material}

We used eight normal cross-bred wolf dogs (age 1-3 yrs, weight $20-25 \mathrm{~kg}$ ). Raw materials included two types of medical-class silicone tubing (Rubber research Institute, Tianjin, China): Type I, which was suitable for the tube-insertion method, and Type II, which was suitable for end-to-end anastomosis. The diameters of the tubes measured 1.2, 1.7, 2.2, and $3.2 \mathrm{~mm}$, respectively (Fig. 1).

\section{Primary Neurodiagnostic Instruments}

We preformed digital angiography DIVAS, (CGR, Paris, France) and TCD ultrasonography (TC-2000, EME Company, West Germany [4MHz]).

\section{Surgical Procedure}

Sodium pentobarbital (20-30 mg/kg) was used to anesthetize all dogs. The frontal parietal approach was undertaken via a 5-cm-long and 4-cm-wide craniectomy that extended to and fully exposed the SSS. Follow-up examinations were conducted by direct angiography: the SSS was punctured, contrast medium $(60 \%$ Conray, $1 \mathrm{ml} / \mathrm{sec}-$ 
ond) was injected, and photographs were continuously obtained. Additionally we performed TCD ultrasonography in which a 4-MHz detecting head was used to scan the SSS directly. Baseline examinations were conducted in all dogs preoperatively and then two dogs each were reexamined at 1, 2, 4, and 8 weeks postoperatively.

To perform the surgical procedure, the operative microscope and vascular microsurgical instruments were used. After opening the dura in a linear fashion on each side of the SSS (1- to 1-cm-long incisions), No. 5-0 silk sutures were sutured through falx cerebrithe at proximal and distal sites to allow ligation later in the procedure. The SSS was occulated using vascular clips at proximal and distal sites and the segment of SSS between the two clips was resected (incisions 8-mm-long resected).

\section{Reconstruction Technique}

The reconstruction method was determined by the width and free length of the SSS.

Tube Insertion. When the width of the SSS was less than $2 \mathrm{~mm}$ or the free length of the SSS was less than 1 $\mathrm{cm}$, a Type I silicone tube (diameter matching the width of the SSS) was used (Fig. 1 upper). The lumen of the silicone tube and SSS were flushed by saline heparinization $(12500 \mathrm{U} / 100 \mathrm{ml})$, one end of the tube was inserted into the proximal end of the SSS, and the other end was inserted into the distal end of the SSS. After removal of the vascular clips, venous flow from the distal to the proximal side of SSS was observed, and both ends of the tubes were fixed using No. 4-0 silk threads (Fig. 2). This technique was performed in six dogs.

End-to-End Anastomosis. When the width of the SSS was greater than $2 \mathrm{~mm}$ and the free length of the SSS was greater than $1 \mathrm{~cm}$, a Type II silicone tube (diameter matching the width of the SSS) was used (Fig. 1 lower). A longer silicone tube was inserted in another selected silicone tube as inner cannula. Both ends of the inner cannula were inserted into the distal and proximal lumina of SSS to ensure that unobstructed SSS blood flow was maintained when the anastomosis was performed. The anastomosis of silicone tube and the distal site of the SSS was first conducted using No. 8-0 silk sutures (Figs. 3 and $4)$; thereafter, anastomosis of the proximal site was performed with the second, fifth, and sixth sutures, after the

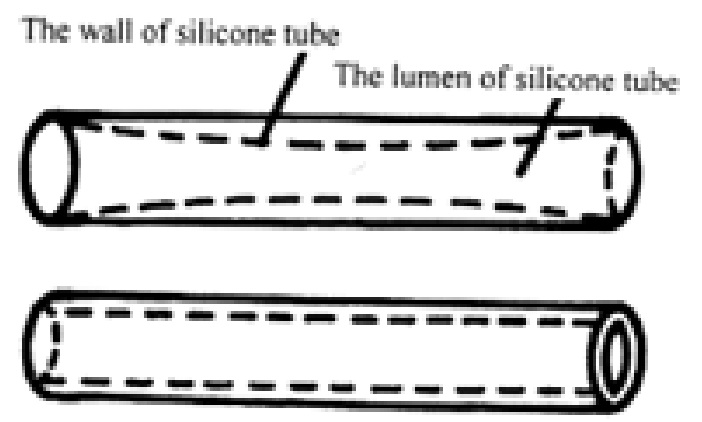

Fig. 1. Diagrams. Upper: A Type 1 silicone tube The wall of the tube is thickest at the center and gradually reduces in size toward both ends, resulting into a crescent-shaped lumen. Lower: A Type II silicone tube. The wall size of the silicone tube is uniform.

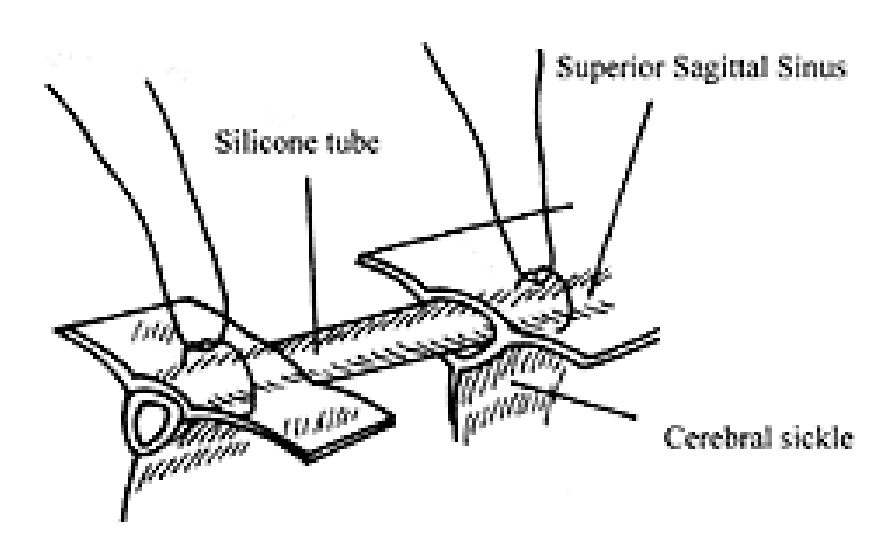

Fig. 2. Diagram showing reconstruction of SSS (tube-insertion method).

inner cannula was removed, and the lumen was flushed with heparinized saline $(12500 \mathrm{U} / 100 \mathrm{ml})$. Both stomata were satisfactory. The silicone tube and SSS were filled with blood, and blood flow was observed. During the anastomosis procedure, the sinus was clamped for a 20- to 30-minute interval and then released to avoid brain edema complications.

\section{Postoperative Course}

The dogs were given aspirin, (500 mg three times a day, ground and mixed with food), and antibiotic therapy was initiated. Abnormal reactions or signs and symptoms of neurological compromise were closely documented. At 1, 4 , and 8 weeks postoperatively, two dogs were killed (one dog was killed at Week 2) and brain biopsy specimens were obtained and sent for histological examination. The grafts were examined under the light and electron microscopes.

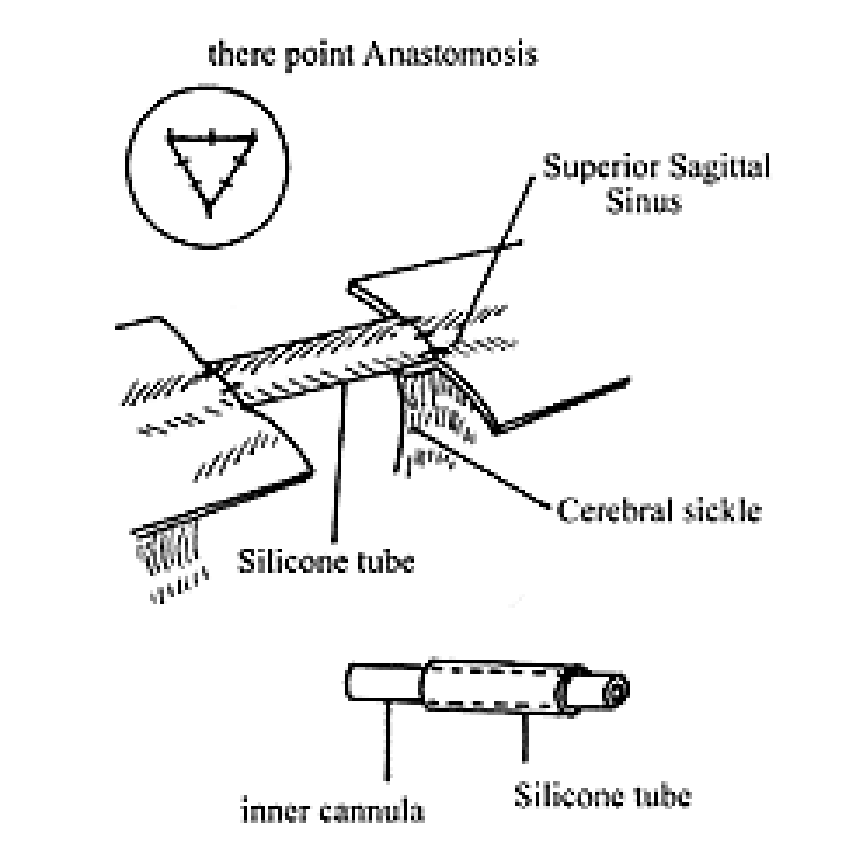

Fig. 3. Diagram demonstrating reconstruction of SSS (end-toend anastomosis method). 


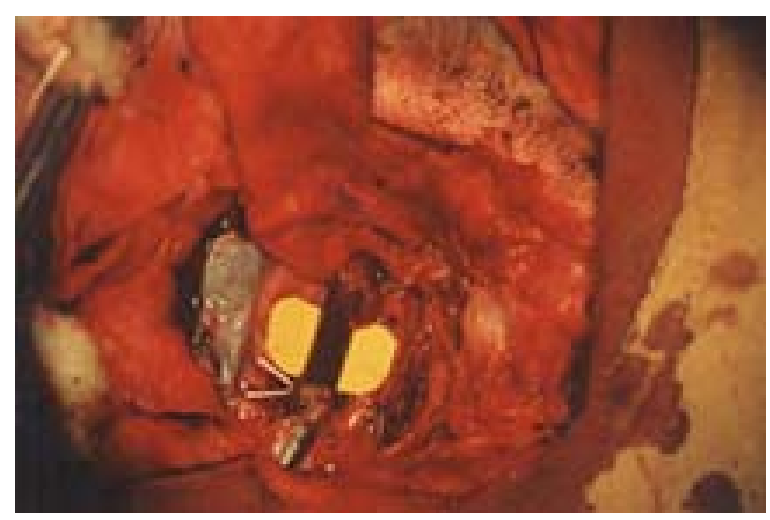

Fig. 4. Photograph demonstrating blood flow through the silicone tube after end-to-end anastomosis.

\section{RESULTS}

\section{Preoperative Examination}

Direct angiography was performed in all eight dogs, and the SSS, transverse sinus, sigmoid sinus, and confluence of sinuses were all clearly visible. In all dogs TCD ultrasonography demonstrated that the SSS was normal.

\section{Postoperative Examination}

In terms of neurological signs and symptoms, all dogs except one developed transient vomiting on the day of surgery (related to the anesthesia); postoperatively no adverse neurological signs and symptoms were demonstrated. During Week 2, one dog escaped from the laboratory.

Direct Angiography. Except for the dog that was lost to follow up, all dogs underwent angiography of the sinus at $1,2,4$, and 8 weeks postoperatively. In five of the seven dogs good SSS patency was demonstrated, and in two patency was occluded during in the 4th week (one underwent tube-insertion reconstruction and the other underwent end-to-end anastomosis reconstruction) (Table 1).

Direct TCD Ultrasonography. In five dogs with patent grafts, postoperative flow signal was the same as that demonstrated (Fig. 5); however, the wave amplitude was lower. The maximum flow rate was 5 to $7 \mathrm{~cm} / \mathrm{second}$ flow (mean $\leq 2 \mathrm{~cm} / \mathrm{second}$ ). Two dogs with obstructed SSSs showed absence of flow signals.

Histological Examination. Before the dogs were killed, brain tissue specimens showed a normal color, with no congestion, no edema, and with normal pulsation. Proliferation of granulation tissue to different degrees was found at different time points postoperatively in the stoma. Of the seven grafts examined, five were patent and the inner surface was smooth with no thrombosis; one in which the tube-insertion method was undertaken was occluded due to improperly tight ligation; one in which the end-toend method performed was thrombotic at the proximal site, but the distal site was patent and the inner surface was smooth with no thrombosis.

Histologically one layer of endothelial cells covered the boundary of silicone tube and SSS at 8 weeks postoperatively, and several new endothelial cells were seen on the surface of lumen of silicone tube. There were infiltrating neutrophils, collagen fibers, and newly formed capillary
TABLE 1

Summary of postoperative SSS angiographic results in eight dogs

\begin{tabular}{clcl}
\hline \hline $\begin{array}{c}\text { Case } \\
\text { No. }\end{array}$ & $\begin{array}{c}\text { Reconstruction } \\
\text { Method }\end{array}$ & $\begin{array}{c}\text { Follow-Up } \\
\text { Time (wks) }\end{array}$ & $\begin{array}{c}\text { Angiographic } \\
\text { SSS Patency }\end{array}$ \\
\hline 1 & tube insertion & 1 & good \\
2 & tube insertion & 4 & partial \\
$3 *$ & anastomosis & 1 & NA \\
4 & anastomosis & 8 & good \\
5 & tube insertion & 4 & occluded \\
6 & tube insertion & 2 & good \\
7 & tube insertion & 2 & good \\
8 & tube insertion & 4 & \\
\hline
\end{tabular}

* Lost to follow up. Abbreviation: NA = not available.

network in the interspace wall of the sinus. Crevicelike small blood vessels were dilated for compensatory collateral circulation. A few foreign-body giant cells were also seen around the SSS. At 8 weeks postoperatively electron microscopy showed proliferated collagen fibers under the endothelium of the SSS close to the silicone tube with glycoprotein deposited among them. Endothelial cells also proliferated. Some were degenerated with larger nuclei, weak color chromatin, invaginated nuclei, bits of heterochromatin that appeared broken, small and irregular nucleoli, medium-amount mitochondria and rough endoplasmic reticula in the cellular plasma, and a small cluster of cellulose deposited on the surface of the endothelium of SSS. The material of the silicone tube was homogeneous with no changes noted.

\section{DISCUSSION}

\section{Necessity of SSS Reconstruction and Use of Silicone Tubing}

Trauma, tumor, thrombosis, and surgery are the main causes of SSS obstruction. This occlusion, especially when acute, provokes a series of life-threatening pathophysiological changes, such as obstruction of venous return, resulting into cerebral edema and increased intracranial pressure. In approximately $4 \%$ of patients sustaining head trauma, dural venous sinus injury also occurs, in most cases involving the SSS. If the SSS is completely resected, end-to-end anastomosis is not possible; however, a tubelike graft, composed of material such as autogenous

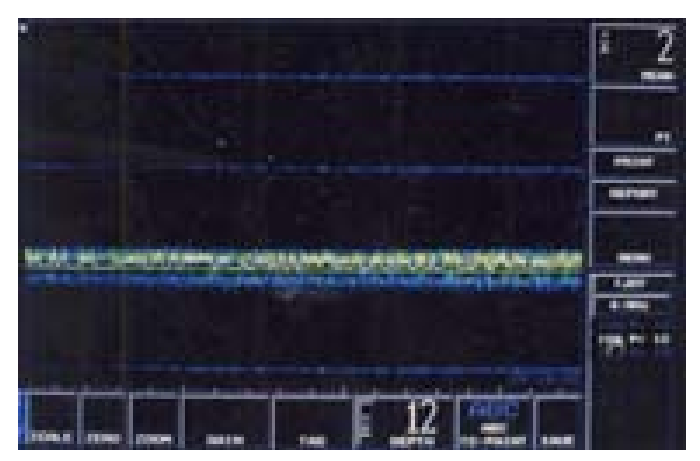

Fig. 5. A TCD ultrasound demonstrating blood flow in the reconstructed SSS after 8 weeks postoperatively. 
veins and artificial blood vessels, may be used to reconstruct the sinus. It is sometimes very difficult to meet the criteria for Simpson Grade I total resection ${ }^{9}$ in cases of parasagittal meningiomas that are located in the middle and posterior two third of the SSS, because the SSS will also require resection. The extent of meningioma resection in this region is closely related to tumor recurrence. Recurrence rates after total resection of meningiomas involving the sagittal sinus wall range from 5 to $9 \%$; those after subtotal resection accompanied by sinus wall excision range from 16 to $17 \%$; after subtotal resection of the tumor only is approximately $29 \%$; and after partial resection of the tumor it is approximately $39 \%$. Liang, et al. ${ }^{4}$ cultured involved dura and found evidence of tumor cell growth beginning on Days 2 to 6 . When the tumor was located on the anterior one third of the SSS, total resection of tumor and involved sinus wall is the best way to prevent recurrence. This is because generally no clinical manifestation will occur when the anterior one third of the SSS and its drainage veins are ligated. Some researchers, however, have found that cerebral parenchymal damage at the different levels occurs in some patients, based on postoperative computerized tomography and magnetic resonance imaging findings. Therefore, it can be noted that proper management of involved SSSs is the key point in preventing parasagittal meningioma recurrence. Similarly, reconstruction of the damaged SSS is necessary to restore its anatomical structural integrity, thereby maintaining normal physiological functioning of the intracranial dural venous sinuses and preventing postoperative complications.

Andrews, et al., ${ }^{1}$ reported that the width of SSS in human beings ranged from 4.3 to $9.9 \mathrm{~mm}$, the height ranged from 3.6 to $6.8 \mathrm{~mm}$, and that it was very suitable for reconstruction. Following the development of microsurgical techniques, intracranial dural venous sinus and related vein reconstruction became possible. Kapp, et al., ${ }^{3}$ used an autogenous great saphenous vein and shunt device to reconstruct the SSS for the first time in 1971. Marks, et al., ${ }^{5}$ performed an SSS bypass operation in 1986. In 1987 Sakaki, et al., ${ }^{8}$ succeeded for the first time in reconstructing cortex veins with silicone tube. Since that time, reconstruction of the SSS and its main drainage veins has occasionally been reported. ${ }^{2,8,10,11}$ At present, the main materials used to repair vessels are the autogenous great saphenous vein, neck superficial veins, and artificial blood vessels such as Dacron and polytetrefluoroethylene, which are suitable for the larger diameter of arterial system, whereas silicone tubing is suitable for dural venous sinuses and veins. Silicone is a form of high polymer with an elastic body, which is formed by inorganic and organic molecules. Its characteristics involve a large energy linkage, stable chemical behavior, nonpoisonous, strong hydrophobicity, and a tolerant biological substance. Compared with other materials, silicone tubes are not thrombogenic, exhibit no tissue-rejection reactions, and have fewer peripheral tissue reactions, less granulation hyperplasia, good compliance, and high patency rate. ${ }^{6,711}$ The feasibility of reconstructing blood vessels (especially dural venous sinuses) with silicone tubing was confirmed by our experiment as well as by other researchers; good re- sults were observed at least within the short term (8 wks), but long-term results require additional research.

\section{Significance of Angiography and TCD Ultrasonography in SSS Reconstruction}

Digital subtraction angiography is the most effective and reliable modality with which to diagnose, treat, and evaluate cerebrovascular diseases in the field of neurosurgery. Our experimental results and those of others indicate that direct angiographic examination of the canine SSS provides better visualization than other studies such as retrograde phlebography and arteriography. This may be because of the numerous collateral circulation systems in the canine SSS and venous system in the base of skull.

In our experiment, direct angiography demonstrated satisfactory visualization. The patency and shape of the SSS and graft were revealed clearly. Direct TCD ultrasonographic visualization of the SSS was also conducted in our study. Although the canine SSS is very thin and the flow rate is low, evolution is still possible by comparing pre- and postoperative flow rates, spectra, and amplitudes. This method is simple, direct, noninvasive, and convenient to repeat.

\section{References}

1. Andrews BT, Dujovny M, Mirchandani HG, et al: Microsurgical anatomy of the venous drainage into the superior sagittal sinus. Neurosurgery 24:514-520, 1989

2. Bederson JB, Eisenberg MB: Resection and replacement of the superior sagittal sinus for treatment of a parasagittal meningioma: technical case report. Neurosurgery 37:1015-1019, 1995

3. Kapp JP, Gielchinsky I, Petty C, et al: An internal shunt for use in the reconstruction of dural venous sinuses. Technical note. $\mathbf{J}$ Neurosurg 35:351-354, 1971

4. Liang Z, Wang M, Pu P: Recurrence problem after total resection of intracranial benign meningioma. Chn J Neurosurg 6: 5-7, 1990

5. Marks SM, Whitwell HL, Lye RH: Recurrence of meningiomas after operation. Surg Neurol 26:436-440, 1986

6. Oka K, Go Y, Kimura H, et al: Obstruction of the superior sagittal sinus caused by parasagittal meningiomas: the role of collateral venous pathways. J Neurosurg 81:520-524, 1994

7. Parker JW, Gaines, RW Jr: Long-term intravenous therapy with use of peripherally inserted silicone-elastomer catheters in orthopaedic patients. J Bone Joint Surg 77:572-577, 1995

8. Sakaki T, Morimoto T, Takemura K, et al: Reconstruction of cerebral cortical veins using silicone tubing. Technical note. J Neurosurg 66:471-473, 1987

9. Simpson D: The recurrence of intracranial meningiomas, after surgical treatment. J Neurol Neurosurg Psychiatry 20:22-39, 1957

10. Steiger HJ, Reulen HJ, Huber P, et al: Radical resection of superior sagittal sinus meningioma with venous interposition graft and reimplantation of the rolandic veins. Case report. Acta Neurochir 100:108-111, 1989

11. Wei X, Li Z, Lin S: Reconstruction of sagittal sinus after total resection of parasagittal meningioma. Chn J Neurosurg 10: 313-315, 1994

Manuscript received December 3, 2001.

Accepted in final form March 25, 2002.

Address reprint requests to: Jingjian Ma, M.D., Ph.D., Department of Neurosurgery, Tianjin Medical University, General Hospital, Tianjin 300052, People's Republic of China. 